Relations industrielles

Industrial Relations

\title{
Ali Dastmalchian, Paul Blyton and Raymond Adamson. The Climate of Workplace Relations
}

\section{Roch Laflamme}

Volume 47, numéro 2, 1992

URI : https://id.erudit.org/iderudit/050781ar

DOI : https://doi.org/10.7202/050781ar

Aller au sommaire du numéro

Éditeur(s)

Département des relations industrielles de l'Université Laval

ISSN

0034-379X (imprimé)

1703-8138 (numérique)

Découvrir la revue

Citer ce compte rendu

Laflamme, R. (1992). Compte rendu de [Ali Dastmalchian, Paul Blyton and

Raymond Adamson. The Climate of Workplace Relations]. Relations industrielles

/ Industrial Relations, 47(2), 383-385. https://doi.org/10.7202/050781ar

Tous droits réservés (C) Département des relations industrielles de l'Université Laval, 1992
Ce document est protégé par la loi sur le droit d'auteur. L’utilisation des services d'Érudit (y compris la reproduction) est assujettie à sa politique d'utilisation que vous pouvez consulter en ligne.

https://apropos.erudit.org/fr/usagers/politique-dutilisation/ 
currently facing the U.S. economy, nor can it be expected to, the advance of employee ownership during the last ten years is truly astonishing, and Rosen and Young are right to point that out. If read widely, as it deserves to be, this book has the potential to dispel much of the confusion and misinformation that currently mars the general understanding of this important concept.

Richard J. LONG

University of Saskatchewan

The Climate of Workplace Relations, by Ali Dastmalchian, Paul Blyton and Raymond Adamson, Routledge, London and New York, 1991, 215 p., ISBN 0-415-03738-7.

Ce volume, rédigé sous la forme d'une thèse ou d'un rapport de recherche, vise à présenter un nouveau modèle des relations du travail où le climat entre les parties peut grandement influencer la gestion des ressources humaines, la structure organisationnelle et les politiques touchant les individus.

Le volume comprend sept chapitres. Le premier chapitre fait un survol du concept de relations industrielles et de celui des théories des organisations. II présente les intentions et le plan de la recherche. Le second chapitre aborde les changements et la dynamique des relations du travail au sein des organisations. Les auteurs présentent les changements dans un contextes où évolue les relations du travail, la structure industrielle, la composition de la main-d'oeuvre et l'organisation syndicale. Tous ces changements conduisent les auteurs à s'interroger sur l'avenir des relations du travail. La diminution du nombre de syndiqués, la précarité des emplois, les nouvelles approches en gestion peuvent-elles réduire l'importance des relations du travail? Les auteurs pensent que les relations du travail demeureront aussi importantes dans l'avenir, cependant, il faudra repenser les mécanismes et créer un climat où les parties pourront échanger et collaborer.

Le troisième chapitre s'attarde au concept de climat. Une revue assez complète des recherches publiées sur le sujet y est présentée. Cependant, certains auteurs importants sont absents: Friendlander et Margulies (1969), Meyer (1969), Prichard et Karasik (1973), Gavin (1975) et Drexler (1977). Les auteurs proposent un modèle d'analyse du climat des relations du travail. Ce modèle s'inspire de l'approche systémique où l'on retrouve comme intrants le contexte organisationnel, la structure, les politiques de ressources humaines et le contexte des relations du travail. La transformation des intrants se résume à l'importance attribuée aux activités et au processus des relations du travail. Les extrants se composent des incidents, du taux de roulement, de l'absentéisme, du succès des négociations, de la perception des syndicats et de la direction face aux relations du travail. Finalement, un processus de rétroaction existe entre les extrants et les intrants. L'approche systémique est très répandue en relations industrielles, néanmoins, l'approche stratégique où l'on tient compte de l'environnement externe et de l'environnement interne en considérant la mission, les valeurs, la culture, les forces et les faiblesses de l'organisation aurait sans doute pu enrichir ce modèle. Ce chapitre contient une note où les auteurs présentent les relations entre le concept de climat organisationnel et celui de 
culture organisationnelle. Ils indiquent que ces relations sont asymétriques, i.e. que chacun des concepts influence l'autre. Donc, même si la culture ne fait pas partie du modèle, une attention particulière lui est portée dans la construction des outils de cueillette de l'information.

Le quatrième chapitre présente le devis de recherche. L'étude réalisée entre juillet 1987 et mars 1988 regroupait 51 entreprises situées en Ontario, au Manitoba, en Saskatchewan et en Alberta. Parmi ces entreprises, 28 oeuvraient dans le secteur manufacturier et 23 dans le secteur des services. La majorité des entreprises regroupait entre 100 et 500 employés. Les années d'exploitation des entreprises variaient entre 13 ans (firmes électroniques) et au-delà de 200 ans (brasseries) avec une moyenne de 63,6 années. Autour de $36 \%$ des entreprises appartenaient aux gouvernements, les autres étaient des entreprises privées dont $30 \%$ offraient des actions au grand public.

A partir du modèle conceptuel d'évaluation du climat de relations du travail présenté au chapitre 3, un protocole d'entrevue ainsi qu'un questionnaire furent réalisés. Pour chacun des indicateurs identifiés, une série de questions étaient élaborées. Les sources d'information vinrent de directions d'entreprise, de gestionnaires, de syndicats ou d'associations d'employés, de documents écrits, de politiques de ressources humaines et de statistiques diverses. Les données furent réunies à l'aide d'entrevues, d'un questionnaire de climat et par la consultation de statistiques concernant le personnel. La recherche visait à administrer le questionnaire du climat à environ $10 \%$ des employés de chacune des entreprises. Au total, 3000 questionnaires furent expédiés et 1686 furent traités. En 1989, cette recherche pris une tournure longitudinale. En effet, les auteurs décidèrent de contacter les directions et les syndicats de 20 entreprises faisant parti de l'échantillon de 1987. De ce nombre, six acceptèrent de participer à nouveau à la recherche. De juillet à novembre 1989 la même cueillette de données qu'en 1987 se réalisa. Sur les 580 questionnaires expédiés, 245 furent traités. II aurait été intéressant que cette étude soit de nature longitudinale dès le départ, ceci aurait contribué à augmenter le nombre d'entreprises participantes à la seconde expérimentation.

Le chapitre 5 présente les résultats de la recherche et les méthodes statistiques retenues pour l'analyse des données. Les chercheurs visaient à tester le modèle en présentant les interrelations entre les variables intrants, celles entre les intrants et le climat et finalement celles entre les résultats par rapport aux attentes exprimées, soient les extrants. L'analyse et l'interprétation des données se feront au chapitre suivant. Au chapitre 6, les auteurs se sont davantage attardés à l'étude longitudinale, ce qui représente 6 organisations et leurs syndicats respectifs. L'analyse des résultats démontre que dans 4 entreprises sur 6 , un changement dans les intrants (contexte organisationnel et structure, contexte des relations du travail et pratiques en gestion des ressources humaines) peut avoir un impact significatif sur le climat de travail et affecter les extrants touchant les relations du travail.

En conclusion, selon les auteurs, le modèle présenté pour évaluer le climat des relations du travail contribue à confirmer les hypothèses de départ. Cependant, il faudra raffiner le modèle à l'avenir. En effet, ce dernier ne semble pas mesurer le degré de conflit ou de rivalité existant au sein des organisations. De plus, ce modèle s'est attardé principalement à la négociation locale laissant 
quelque peu de côté les effets de la négociation dans un contexte sectoriel ou multi-employeurs. Également, la comparaison des conditions du marché du travail fut difficile à cause d'une trop grande hétérogénéité des entreprises faisant partie de l'échantillon.

Même avec ces lacunes, ce modèle fait office de pionnier dans ce domaine et peut servir de balise à d'autres recherches.

Roch Laflamme

Université Laval

Interactive Human Resource Management and Strategic Planning, par Cynthia A. Lengnick-Hall et Mark L. Lengnick-Hall, New York, Quorum Books, 1990, 196 p., ISBN 0-89930-502-4

Une dizaine d'années se sont déjà écoulées depuis que l'idée d'aborder la gestion des ressources humaines selon une perspective stratégique a été proposée aux États-Unis (Dyer 1983). En dix ans, ce courant de recherche a réussi à attirer l'attention des chercheurs, comme en témoignent les nombreux écrits - parfois empiriques, parfois spéculatifs - sur le sujet (Butler, Ferris et Napier 1991). Le temps était donc venu de proposer une synthèse de ces travaux, ce qui s'est produit en France en 1988 (Besseyre des Horts), au Québec en 1991 (Wils, Le Louarn et Guérin) et aux États-Unis également en 1991 avec la parution du livre de Lengnick-Hall et Lengnick-Hall. Contrairement au livre américain de Fombrun, Tichy et Devanna (1984), ce livre n'est pas un recueil de textes mais une synthèse originale des écrits. La recension de cet ouvrage commencera par un résumé des différents chapitres afin de mettre en relief quelques interrogations que cette lecture nous a inspirées et se terminera par quelques commentaires plus généraux.

Le livre de Lengnick-Hall et Lengnick-Hall est composé de sept chapitres. Le premier chapitre intitulé Defining Strategic Human Resource Management ne propose pas vraiment une définition de la gestion stratégique des ressources humaines, mais explicite plutôt des postulats et des prescriptions. Par postulat, nous entendons des affirmations du genre "les ressources humaines sont une ressource critique pour bâtir un avantage compétitif". Si une telle affirmation était toujours vraie, on peut se demander pourquoi tant de dirigeants d'entreprise ne se servent pas davantage de cette ressource pour assurer leur survie. C'est alors que les auteurs ont recours à des prescriptions en précisant que cet avantage ne peut se concrétiser que dans la mesure où plusieurs prescriptions sont suivies. A titre d'exemple, une de leurs prescriptions se lit comme suit: Human resource management should be an integral part of the strategic planning processes of a firm (page 4). Somme toute, ce premier chapitre est une profession de foi en l'école du "choix stratégique" qui reconnait l'importance des décideurs. Le seul reproche qui pourrait leur être adressé est de ne pas avoir pris le temps de bien définir la gestion stratégique des ressources humaines et de proposer des postulats et des prescriptions sans en discuter leur bien-fondé.

Avant d'aborder le lien entre la ressource humaine et la gestion stratégique, il est logique de commencer par comprendre le concept de stratégie, 